\title{
Random Access Preamble Generation with Low Storage for LTE Networks
}

\author{
Pei-Zhe XIN ${ }^{1}$, Yu-Qi LV ${ }^{2, a}$, Lan SU ${ }^{3}$ and Yu-Dong WANG \\ ${ }^{1}$ State Power Economic Research Institute, Beijing 100052, China, 10653224309@qq.com \\ ${ }^{2}$ University of Electronic Science and Technology of China, Chengdu, China \\ ${ }^{3}$ State Power Economic Research Institute, Beijing 100052, China
}

\begin{abstract}
Long Term Evolution (LTE) random access preambles are generated from cyclically shifted Zadoff-Chu(ZC) sequences of prime length by using so-called Discrete Fourier Transformation-Spread-Orthogonal Frequency Division Multiplexing (DFT-S-OFDM) modulation. In this paper we propose a novel preamble generation to reduce the storage and complexity of cyclically shifted ZC sequences' Discrete Fourier Transformation (DFT). The proposed preamble generation allows the DFT of cyclically shifted ZC sequences to rely only on several constant factors and the elements of non-cyclically shifted ZC sequences which have already been generated. This preamble generation merely requires the storage of several constant factors, while avoids large storage and complex computation of complex exponential function.
\end{abstract}

\section{Introduction}

As the rapid development of the communication technology and the microelectronic technology, the electronic power system develops gradually towards informatization and intellectualization. The electric energy data acquire system is an important part of smart grid which covers the power users and electrical information. Stable and reliable communication network are the guarantee of energy data acquisition. Compared with the optical fiber network, wireless communication network has a lower cost, and can directly use of the existing mobile communication network to establish connections quickly.

Electric energy data acquire system consists of three parts: master station, communication network and collectors. Its structure is shown in Fig. 1. Master station is the center of electric energy data acquire system, which is responsible for controlling the data acquire terminals, processing and sharing the information of electric energy, and offering the data interface to the services of electric power department and the marketing applications. Communication network provides a communication channel to connect data acquire terminals and master station.

Next taking the LTE communication system for an example, the paper illustrates the advantage of the electric energy data acquire system. The random access channel (RACH) is a common uplink channel in LTE communication systems used by the mobile user equipment (UE) to establish the initial access and uplink synchronization to a base station (Node B) [1]-[4]. The random access (RA) preambles transmitted from the UE are generated from cyclically shifted Zadoff-Chu sequences,

a Corresponding author: lyq-tiantian@139.com 
owing to their good correlation properties [5]. In the generation of the random access preambles, first we need to perform an $N$-point discrete fourier transform of a cyclically shifted ZC sequences defined by the root index $u$ and the cyclic shift $C_{v}$. However, large storage and complex computation of complex exponential function in DFT cannot meet the mobile transceivers' storage and computational requirements.

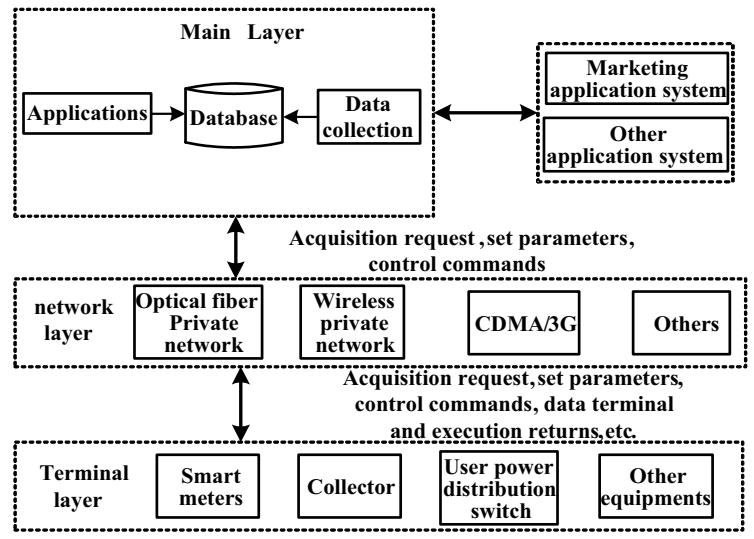

Figure 1. Structure of electric energy data acquire system

To reduce the storage and complexity of cyclically shifted ZC sequences' discrete fourier transform, several effective work has been done. CHARLES M. RADER expressed a fast fourier transform (FFT) algorithm of prime length $M$ as a fast convolution [6-7]. But the computation of three FFTs of highly composite length $N>M$ is still required. Then the efficient discrete fourier transform structures more suitable for applications of ZC sequences have been derived [8]. Nevertheless, the discrete fourier transform structures in [8] cannot always be used to reduce the number of operations in the transmitter of long term evolution user equipment, as the transmitted random access (RA) preamble can be a cyclically shifted version of the basic ZC sequences. In addition, this discrete fourier transform structures cannot avoid large storage and complex computation of complex exponential function.

In this paper, we propose the discrete fourier transform of cyclically shifted ZC sequences of prime length depends only on several constant factors and the elements of the non-cyclically-shifted ZC sequence which has already been generated. The fact allows us to reduce the number of arithmetic operations and the storage of complex exponential function for computing the discrete fourier transform of a cyclically shifted ZC sequence of prime length.

\section{Definition and previous DFT of preamble sequences}

In this paper, we propose the discrete

\subsection{Definition}

The $u^{\text {th }}$ root Zadoff-Chu sequence of prime length $N_{\mathrm{ZC}}$ is defined as [5]

$$
x_{u}(n)=e^{-j \frac{\pi u n(n+1)}{N_{\mathrm{ZC}}}}, \quad 0 \leq n \leq N_{\mathrm{ZC}}-1
$$

where the root index $u$ is an integer less than the prime $N_{\mathrm{ZC}}$. From the $u^{\text {th }}$ root Zadoff-Chu sequence, random access preambles with zero correlation zones of length $N_{\mathrm{CS}}-1$ are defined by cyclic shifts according to [5] 


$$
x_{u, v}(n)=x_{u}\left(\left(n+C_{v}\right) \bmod N_{z C}\right)
$$

where the cyclic shift $C_{v}$ is equal to $v N_{\mathrm{CS}}$, for unrestricted sets. [8]:

In LTE, the uplink random access preamble is defined by a complex baseband signal of the form

$$
s(k)=\sum_{m=0}^{N_{\mathrm{ZC}}-1} \sum_{n=0}^{N_{\mathrm{ZC}}-1} x_{u, v}(n) e^{-j \frac{2 \pi n m}{N_{\mathrm{ZC}}}} \cdot e^{j \frac{2 \pi\left(m+m_{0}\right) k}{L}}
$$

where $-N_{\mathrm{CP}} \leq k<L$ and $N_{\mathrm{ZC}}=839, L=24576 . N_{\mathrm{CP}}$ is the variable cyclic prefix length. The above formula is the discrete time expression of the formula given in [5] where a number of random access protocol parameters have been condensed into a single $m_{0}$ for brevity. In the generation of such RA preambles, the first step is to perform a $N$-point discrete Fourier transform of a cyclically shifted ZC sequence by the root index $u$ and the cyclic shift $C_{v}$.

\subsection{Previous DFT of preamble sequences}

In practice, the DFT is almost always implemented as a FFT, which allows a reduction in the number of arithmetic operations from approximately $N^{2}$ to $N \log _{2} N$ [9]. Considering the special properties of ZC sequences, an attempt to reduce the computation complexity of ZC sequences' DFT has been done in [8]. The DFT of the $u^{\text {th }}$ root ZC sequence $x_{u}(n)$ of prime length $N_{\mathrm{ZC}}$ is equal to a conjugated and time-scaled version of $x_{u}(n)$, up to a constant factor:

$$
X_{u}(k)=x_{u}^{*}\left[u^{-1} k\right] X_{u}(0)
$$

where '*' denotes complex conjugation, and $u^{-1}=1 / u$ is the modular multiplicative inverse of $u$, i.e. an integer such that $u u^{-1}=1 \bmod N_{\mathrm{ZC}}$.

By using the DFT properties of cyclically shifted sequences, the DFT $X_{u, v}(k)$ of cyclically shifted ZC sequence $x_{u, v}(n)$ can be expressed as

$$
X_{u, v}(k)=X_{u}(k) e^{j \frac{2 \pi k C_{v}}{N_{z c}}}
$$

By applying (4) to (5), we obtain

$$
X_{u, v}(k)=x_{u}^{*}\left(u^{-1} k\right) X_{u}(0) e^{j \frac{2 \pi k C_{v}}{N_{z c}}}
$$

It is obvious that (6) cannot avoids complex computation of complex exponential function in computing the DFT of cyclically shifted ZC sequences. In addition, $\left(N_{\mathrm{ZC}}-1\right)$ complex values of $X_{u}(0)$ should be prestored in memory, since there are $\left(N_{\mathrm{ZC}^{-}}-1\right)$ root Zadoff-Chu sequences that can be transmitted from the mobile transceivers [5].

\section{Proposed preamble generation}

To avoid complex computation of complex exponential function and obtain the structure of an efficient DFT of a cyclically shifted ZC sequence, (6) should be transformed into another form that includes no extra complex exponential functions.

By utilizing the definition of the $u^{\text {th }}$ root Zadoff-Chu sequence of prime length $N_{\mathrm{ZC}}$, we obtain that 


$$
\begin{aligned}
x_{u}^{*}\left(u^{-1} k\right) e^{j \frac{2 \pi k C_{v}}{N_{z c}}} & =e^{j \frac{\pi u\left[u^{-1} k\left(u^{-1} k+1\right)\right]}{N_{z c}}} e^{j \frac{\pi u\left(2 u^{-1} k C_{v}\right)}{N_{z c}}} \\
& =e^{j \frac{\pi u\left[\left(u^{-1} k+C_{v}\right)\left(u^{-1} k+C_{v}+1\right)-C_{v}\left(C_{v}+1\right)\right]}{N_{z c}}} \\
& =e^{j \frac{\pi u\left[\left(u^{-1} k+C_{v}\right)\left(u^{-1} k+C_{v}+1\right)\right]}{N_{z c}}} e^{-j \frac{\pi u C_{v}\left(C_{v}+1\right)}{N_{z c}}} \\
& =x_{u}^{*}\left(u^{-1} k+C_{v}\right) x_{u}\left(C_{v}\right)
\end{aligned}
$$

In the derivation of (7), the periodicity of the root $\mathrm{ZC}$ sequences has been applied. It is easily verified that $x_{u}(n)$ is periodic with prime $N_{\mathrm{ZC}}$. After substituting (7) into (6), it can be seen that

$$
X_{u, v}(k)=x_{u}^{*}\left(u^{-1} k+C_{v}\right) x_{u}\left(C_{v}\right) X_{u}(0)
$$

Through observing the form of $X_{u}(0)$, we find that $X_{u}(0)$ can be expressed as

$$
\begin{aligned}
X_{u}(0) & =\sum_{n=0}^{N_{Z C}-1} e^{-j \frac{\pi u n(n+1)\left(N_{Z C}+1\right)}{N_{Z C}}} \\
& =\sum_{n=0}^{N_{Z C}-1} e^{-j \frac{2 \pi u \alpha n(n+1)\left(N_{Z C}+1\right)}{N_{Z C}}} \\
& =\sum_{n=0}^{N_{Z C}-1} e^{-j \frac{2 \pi u \alpha\left[(n+\alpha)^{2}-\alpha^{2}\right]}{N_{Z C}}} \\
& =e^{j \frac{2 \pi u \alpha^{3}}{N_{Z C}}} \sum_{n=0}^{N_{Z C}-1} e^{-j \frac{2 \pi u \alpha(n+\alpha)^{2}}{N_{Z C}}} \\
& =e^{j \frac{2 \pi u \alpha^{3}}{N_{Z C}}} G\left(u \alpha, N_{Z C}\right)^{*}
\end{aligned}
$$

where $G(m, L)^{*}$ is the complex conjugate of the quadratic Gauss sum [9]

$$
G(m, L)=\sum_{k=0}^{L-1} e^{j \frac{2 \pi m k^{2}}{L}}
$$

and $\alpha=\left(N_{\mathrm{ZC}}+1\right) / 2$ is an integer for odd prime $N_{\mathrm{ZC}}$. Considering prime $N_{\mathrm{ZC}}$ and $\left(u \alpha, N_{\mathrm{ZC}}\right)=1, G(u \alpha$, $\left.N_{\mathrm{ZC}}\right)^{*}$ can be expressed as

$$
\begin{aligned}
G\left(u \alpha, N_{Z C}\right)^{*} & =\left(\frac{u \alpha}{N_{Z C}}\right) G\left(1, N_{Z C}\right)^{*} \\
& =\left(\frac{u}{N_{Z C}}\right)\left(\frac{\alpha}{N_{Z C}}\right) G\left(1, N_{Z C}\right)^{*}
\end{aligned}
$$

where $\left(u / N_{\mathrm{ZC}}\right)$ and $\left(\alpha / N_{\mathrm{ZC}}\right)$ denote Legendre symbols [10]. And if $u \neq 0 \bmod \mathrm{N}_{\mathrm{ZC}}$, then the values of the Legendre symbol $\left(u / N_{\mathrm{ZC}}\right)$ are 1 or -1 . From (10) and (11), it can be seen that

$$
X_{1}(0)=e^{j \frac{2 \pi \alpha^{3}}{N_{Z C}}}\left(\frac{\alpha}{N_{Z C}}\right) G\left(1, N_{Z C}\right)^{*}
$$

Thus by applying (11) and (12) to (10), we obtain 


$$
X_{u}(0)=e^{j \frac{2 \pi(u-1) \alpha^{3}}{N_{Z C}}}\left(\frac{u}{N_{Z C}}\right) X_{1}(0)
$$

To avoid complex computation of the complex exponential function $e^{j \frac{2 \pi(u-1) \alpha^{3}}{N_{Z C}}}$, (13) should be simplified further as

$$
\begin{aligned}
e^{j \frac{2 \pi(u-1) \alpha^{3}}{N_{Z C}}} & =e^{j \frac{\pi(u-1)\left(1-N_{Z C}\right) \alpha^{2}}{N_{Z C}}} \\
& =e^{-j \frac{2 \pi(u-1) \beta \alpha}{N_{Z C}}\left(\frac{N_{Z C}}{2}+\frac{1}{2}\right)} \\
& =e^{-j \frac{\pi(u-1) \beta \alpha}{N_{Z C}}} \\
& =x_{u-1}(\beta)
\end{aligned}
$$

where $\beta=\left(N_{\mathrm{ZC}^{-}}-1\right) / 2$. And then substituting (13) and (14) into (8), the DFT of cyclically shifted ZC sequences can be expressed as

$$
X_{u, v}(k)=\left(\frac{u}{N_{Z C}}\right) x_{u}^{*}\left(u^{-1} k+C_{v}\right) x_{u}\left(C_{v}\right) x_{u-1}(\beta) X_{1}(0)
$$

To simplify the computation of (15), the constant factors can be prestored in memory. $\left(N_{\mathrm{ZC}}-1\right)$ and $2 M$ bits are needed to prestore the Legendre symbol $\left(u / N_{\mathrm{ZC}}\right)$ and the complex value $X_{1}(0)$ respectively if real and imaginary parts art $M$ bits. Therefore $\left(N_{\mathrm{ZC}}-1+2 M\right)$ bits are needed to prestore the constant factors in memory.

\section{Storage and complexity analysis}

From (15) it follows that, for a given root $u$ and the cyclic shift $C_{v}$ of the ZC sequence of prime length $N_{\mathrm{ZC}}$, the corresponding DFT is cyclically delayed and then cyclically sampled complex conjugated version of already generated basic ZC sequences, multiplied by a constant, which consists of four factors: the $\left(C_{v}+1\right)^{\text {th }}$ element of the $u^{\text {th }}$ root ZC sequence, the $(\beta+1)^{\text {th }}$ element of the $(u-1)^{\text {th }}$ root ZC sequence, the sum of all the elements of the $1^{\text {th }}$ root $Z C$ sequence, and the Legendre symbol. Some constant factors, like the sum and the Legendre symbol, can be prestored in memory to accelerate the generation of LTE random access preambles. For the previous DFT of preamble sequences, $\left(N_{\mathrm{ZC}}-1\right)$ complex values of $X_{u}(0)$ and $N_{\mathrm{ZC}}$ complex values of $\exp \left(j 2 \pi k C_{v} / N_{\mathrm{ZC}}\right)$ should be prestored, while the proposed DFT merely requires the storage of $\left(N_{\mathrm{ZC}}-1\right)$ binary values of the Legendre symbol and one complex values $X_{1}(0)$.

The table of storage and complexity analysis between the previous and proposed DFT of preamble sequences goes as follows.

Table 1. Storage and Complexity Analysis

\begin{tabular}{|c|c|c|}
\hline & $\begin{array}{c}\text { Storage } \\
\text { (bit) }\end{array}$ & $\begin{array}{c}\text { Complexity } \\
\text { (complex } \\
\text { multiplication) }\end{array}$ \\
\hline $\begin{array}{c}\text { Previous } \\
\text { DFT }\end{array}$ & $\left(N_{\mathrm{ZC}}-1\right) \cdot 2 M+N_{\mathrm{ZC}} \cdot 2 M$ & $N_{\mathrm{ZC}}+1$ \\
\hline $\begin{array}{c}\text { Proposed } \\
\text { DFT }\end{array}$ & $N_{\mathrm{ZC}}-1+2 M$ & $N_{\mathrm{ZC}}+3$ \\
\hline
\end{tabular}

Suppose $M$ bits are required for storing the real or imaginary of one complex. 
In the generation of random access preambles for LTE systems, the random access preamble sequence length $N_{\mathrm{ZC}}$ is 839 when preamble formats $0 \sim 3$ are in application [5]. And suppose $M$ is equal to 12 . The different storage requirement of the above two kinds of preamble generation is showed in the following Fig.2.

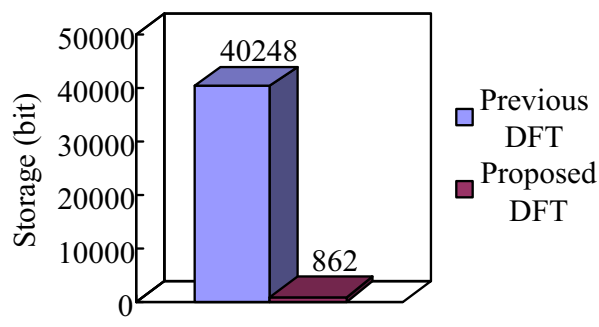

Figure 2. The storage requirement of the previous and proposed DFT of preamble sequences

From the above table and figure it follows that, although proposed DFT of preambles requires two more complex multiplications than the previous one, it reduces $\left(N_{\mathrm{ZC}}-1\right) \cdot 2 M+\left(N_{\mathrm{ZC}}-1\right) \cdot(2 M-1)$ bits storage, which is very helpful for hardware implementation of preamble generation.

\section{Conclusion}

Random access preamble generation of low storage for LTE systems is presented. This preamble generation allows the DFT of cyclically shifted ZC sequences to depend only on several constant factors and the elements of already generated non-cyclically-shifted ZC sequences. In addition, large storage and complex computation of complex exponential function are replaced by prestoring several constant factors, which is significant for hardware implementation of LTE RA preamble generation.

\section{Acknowledgment}

The paper is sponsored by the science and technology program of the State Grid Corporation of China (No. SGTYHT/15-JS-191).

\section{References}

1. Mohammad M. Mansour, "Optimized Architecture for Computing Zadoff-Chu Sequences with Application to LTE," in Global Telecommunications Conference, 2009, pp. 1-6.

2. Yang Wen, Wei Huang, and Zhongpei Zhang, "CAZAC sequence and its application in LTE random access," in Information Theory Workshop, Oct. 2006, pp. 544-547.

3. B. M. Popovic, and O. Mauritz, "Random Access Preambles for Evolved UTRA Cellular System," Spread Spectrum Techniques and Applications, 2006 IEEE Ninth International Symposium on. Aug. 2006, pp. 488-492

4. Mehdi Aminjoo, Fredrik Gunnarsson, and Filip Andren, "3GPP LTE Random Access Channel Self-Optimization”, Proc. IEEE, pp.2784-2793, January 2014.

5. 3GPP TS 36.211, "Evolved Universal Terrestrial Radio Access (E-UTRA); Physical Channels and Modulation," Mar. 2010.

6. Charles M. Rader, "Discrete Fourier transforms when the number of data samples is prime," Proc. IEEE, vol. 56, no. 6, pp. 1107-1108, June 1968.

7. Arun C.A. , P. Prakasam, "Design of high speed FFT algorithm For OFDM technique", Proc. IEEE, October 2016.

8. S. Beyme and C. Leung, "Efficient computation of DFT of Zadoff-Chu sequences," Electron. Lett., vol. 45, no. 9, pp. 461-463, Apr. 2009 
9. John G. Proakis, and Dimitris G. Manolakis, Digital Signal Processing: Principles, Algorithms and Applications, Fourth Edition. June 2007, pp. 379-399.

10. B. C. Berndt, R. J. Evans, and K. S. Willias, Gauss and Jacobi sums. 1998, pp. 7-28.

11. Godfey Harold Hardy, Edward Maitland Wright, and Joseph H. Silvevman, An introduction to the theory of numbers. Mar. 2007, pp. 79-85.

12. Min Hua, Mao Wang, and Kristo Wenjie Yang, "Analysis of the Frequency Offset Effect on Zadoff-Chu Sequence Timing Performance”, Proc. IEEE, pp. 4024-4039, October 2014. 